\author{
T. Nakonechnaya \\ Oles Honchar Dnipro National University
}

\title{
THE CYCLIC INEQUALITY OF N.P. KORNEICHUK AND IT'S GENERALIZATION
}

In this paper there is given a generalization of well-known cyclic inequality of N.P. Korneichuk on the case of $\boldsymbol{n}$ independent variables. This result is of independent interest and can be used to obtain estimated results of splines-approximation in classes with bounded modulus of continuity.

Key words: cyclic inequality, extreme problem, Lagrange principle.

Наведена стаття містить узагальнення відомої циклічної нерівності Н.П. Корнійчука на випадок $n$ незалежних змінних. Отриманий результат має також самостійний інтерес та його можна застосувати для отримання оціночних результатів сплайн-апроксимації на класах функцій з обмеженим модулем неперервності.

Ключові слова: циклічна нерівність, екстремальна задача, принцип Лагранжу.

В данной статье приводится обобщение известного циклического неравенства Н.П. Корнейчука на случай $n$ независимых переменных. Данный результат представляет самостоятельный интерес и может быть использован для получения оценочных результатов сплайн-аппроксимации на классах с ограниченных модулем непрерывности.

Ключевые слова: циклическое неравенство, экстремальная задача, принцип Лагранжа.

Introduction. N.P. Korneichuk in his monograph "Splines in approximation theory" noted the advantages of splines over other approximation methods. Two aspects can be pointed out in which these advantages have been most convincing:

1) Interpolation splines in a number of important cases provide the minimum possible approximation error on the class of functions. Interpolation polynomials do not provide even the best order.

2) Splines - the apparatus is more convenient than polynomials from a computational or even practical point of view.

The main attention in the fifth chapter of his monograph, N.P. Korneichuk focused on issues related to estimating the error of spline-interpolation through the modulus of continuity $\omega(\delta)$. In the same chapter, an upper bound was obtained for the approximation of piecewise constant functions on a class $H^{\omega}$. To establish this estimate, N.P. Korneichuk had to prove the following elementary inequality. We cite this inequality together with the proof.

Lemma (N.P. Korneichuk). For all $u \geq 0$ and $0<p \leq 3$ we have the inequality

$$
2^{p}\left(u^{p}+u\right) \leq(1+u)^{p+1}
$$

which with $p>3$ and some $u>0$ is no longer fulfilled.

(C) Nakonechnaya T., 2019 
Really, let us consider the function

$$
\eta(u, p)=(1+u)^{p+1}-2^{p}\left(u^{p}+u\right), u \geq 0, p>0,
$$

and we'll show that

$$
\eta(u, p) \geq 0, u \geq 0,3 \geq p>0 .
$$

Assuming $u>0$ (with $u=0$ inequality (2) is obvious), we note that $\eta(u, p)=u^{p+1} \eta\left(\frac{1}{u}, p\right)$,

and therefore it is sufficient to prove non-negativity $\eta(u, p)$ with $u \geq 1$ and $0<p \leq 3$.

Thus

$$
\eta(1, p)=\eta_{u}^{\prime}(1, p)=0, \lim _{u \rightarrow \infty} \eta(u, p)=+\infty, p>0,
$$

then necessary inequality will be established if we show that for $u>1$ and $0<p<3$

$$
\eta_{u u}^{\prime \prime}(u, p)>0
$$

But

$$
\eta_{u u}^{\prime \prime}(u, p)=p\left[(p+1)(1+u)^{p-1}-2^{p}(p-1) u^{p-2}\right]
$$

and in the case $0<p \leq 1$ everything is trivial. If $1<p<3$, then (3) follows from fact

$$
\eta_{u u}^{\prime \prime}(1, p)=p 2^{p-1}(3-p)>0
$$

and, besides, for $u \geq 1$ and $1<p<3$

$$
\frac{\partial^{3} \eta(u, p)}{\partial u^{3}}=p(p-1)\left[(p+1)(1+u)^{p-2}-2^{p}(p-2) u^{p-3}\right]>0 .
$$

Last inequality at $1<p \leq 2$ is obvious, and for $u \geq 1$ and $2<p<3$ follows from the relation

$$
(1+p)(1+u)^{p-2}>(1+p) 2^{p-2}>2^{p}(p-2)>2^{p}(p-2) u^{p-3} .
$$

Relation (2), and with it the first part of the statement of lemma, is proved. If $p>3$, then $\eta_{u u}^{\prime \prime}(u, p)<0$ and in the neighborhood of the line $u=1$ function $\eta(u, p)$ takes negative values.

We reformulate this inequality in a more convenient form for us; for this, put $u=x / y$, where $x>0, y>0$.

Lemma 1. For all $x \geq 0, y \geq 0$ and $0<p \leq 3$ we have the inequality

$$
(x+y)^{p+1} \geq 2^{p}\left(x y^{p}+y x^{p}\right),
$$

which with $p>3$ and some $x>0, y>0$ is no longer fulfilled.

The main results. This article summarizes the inequality of N.P. Korneichuk in the case of $n$ independent variables. The following theorem will be true. 
Theorem 1. For all $x_{i}>0(i=1,2, \ldots n), n \in N, n \geq 2$ and $q \in\left(0 ; \frac{n+1}{n-1}\right)$ the next inequality will be true

$$
\left(\frac{1}{n} \sum_{k=1}^{n} x_{k}\right)^{q+1} \geq \frac{1}{n} \sum_{k=1}^{n} x_{k}^{q} \frac{1}{n-1}\left(\sum_{i=1}^{n} x_{i}-x_{k}\right),
$$

which with $q>\frac{n+1}{n-1}$ and some $x_{i}>0(i=1,2, \ldots n)$ is no longer fulfilled. If $q=\frac{n+1}{n-1}$, then inequality (5) holds only for the values $n=2,3$.

Proof. We'll obtain the decision of inequality (5) as a solution to the extreme problem

$$
\left(\frac{1}{n} \sum_{k=1}^{n} x_{k}\right)^{q+1} \rightarrow \inf , q>0, x_{k}>0,(1 \leq k \leq n)
$$

under the following condition

$$
\frac{1}{n} \sum_{k=1}^{n} x_{k}^{q} \frac{\left(\sum_{i=1}^{n} x_{i}-x_{k}\right)}{n-1}-1=0 .
$$

The set of admissible elements is compact; the functional is continuous, which means that by Weierstrass's theorem a solution to the problem exists. First, let the $0<q<\frac{n+1}{n-1}$. Then Lagrange Function have the form:

$$
L=\lambda_{0}\left(\frac{1}{n} \sum_{k=1}^{n} x_{k}\right)^{q+1}+\lambda_{1}\left(\frac{1}{n(n-1)} \sum_{k=1}^{n} x_{k}^{q}\left(\sum_{i=1}^{n} x_{i}-x_{k}\right)-1\right)
$$

The necessary condition for the existence of an extremum is as follows:

$$
\begin{gathered}
\frac{\partial L}{\partial x_{k}}=0,(k=1,2, \ldots . n) \Leftrightarrow \\
\lambda_{0} \frac{q+1}{n}\left(\frac{1}{n} \sum_{k=1}^{n} x_{k}\right)^{q}+\frac{\lambda_{1}}{n(n-1)}\left(q x_{k}^{q-1}\left(\sum_{i=1}^{n} x_{i}-x_{k}\right)+\sum_{i=1}^{n} x_{i}^{q}-x_{k}^{q}\right)=0 .
\end{gathered}
$$

If $\lambda_{0}=0$ then and $\lambda_{1}=0$ that contradicts the Lagrange principle. Put $\lambda_{0}=1$. Subtracting from all subsequent equations the first equation of system (4), we obtain the following system: 


$$
\left\{\begin{array}{l}
\lambda_{0} \frac{q+1}{n}\left(\frac{1}{n} \sum_{k=1}^{n} x_{k}\right)^{q}+\frac{\lambda_{1}}{n(n-1)}\left(q x_{1}^{q-1}\left(\sum_{i=1}^{n} x_{i}-x_{1}\right)+\sum_{i=1}^{n} x_{i}^{q}-x_{1}^{q}\right)=0, \\
\frac{\lambda_{1}}{n(n-1)}\left(q \sum_{i=1}^{n} x_{i}\left(x_{k}^{q-1}-x_{1}^{q-1}\right)-(q+1)\left(x_{k}^{q}-x_{1}^{q}\right)\right)=0, \quad k=2,3, \ldots n .
\end{array}\right.
$$

It is clear that the solution to this system has the form $x_{k}=x_{1}(k=2,3, \ldots n)$. According to condition (6), we obtain, that $x_{1}^{q+1}=1$, hence, $x_{1}=1$ and $\lambda_{1}=-1$. Thus, the point $N(1,1, \ldots .1,-1)$ is the point of the possible minimum of the Lagrange function, and the point $\hat{x}=(1,1, \ldots, 1)$ will be a valid element. Now it is necessary to verify that sufficient conditions are satisfied, that is, to prove that the inequality $\left.d^{2} L\right|_{N}>0$ holds for $q \in\left(0 ; \frac{n+1}{n-1}\right)$. Calculating the second-order derivatives of the Lagrange function, we obtain:

$$
\begin{aligned}
& \frac{\partial^{2} L}{\partial x_{k}^{2}}=\frac{q(q+1)}{n^{2}}\left(\frac{1}{n} \sum_{k=1}^{n} x_{k}\right)^{q-1}+\frac{\lambda_{1} q(q-1)}{n(n-1)} x_{k}^{q-2} \sum_{i=1}^{n} x_{i}-\frac{\lambda_{1} q(q-1)}{n(n-1)} x_{k}^{q-1},(k=1,2, \ldots . . n) \\
& \frac{\partial^{2} L}{\partial x_{k} x_{j}}=\frac{q(q+1)}{n^{2}}\left(\frac{1}{n} \sum_{k=1}^{n} x_{k}\right)^{q-1}+\frac{\lambda_{1} q}{n(n-1)}\left(x_{k}^{q-1}+x_{j}^{q-1}\right), \quad k, j=1,2, \ldots n ; \quad k \neq j .
\end{aligned}
$$

Consequently,

$$
\begin{aligned}
& \left.\frac{\partial^{2} L}{\partial x_{k}^{2}}\right|_{N}=\frac{(n+1) q}{n^{2}}\left(1-q \frac{n-1}{n+1}\right), \quad k=1,2, \ldots n \\
& \left.\frac{\partial^{2} L}{\partial x_{k} \partial x_{j}}\right|_{N}=-\frac{(n+1) q}{n^{2}}\left(1-q \frac{n-1}{n+1}\right), \quad k, j=1,2, \ldots n, \quad k \neq j .
\end{aligned}
$$

Thus, we arrive at the equality

$$
\left.d^{2} L\right|_{N}=\frac{(n+1) q}{n^{2}}\left(1-q \frac{n-1}{n+1}\right)\left(2 \sum_{k=1}^{n} d x_{k}^{2}-\left(\sum_{k=1}^{n} d x_{k}\right)^{2}\right) .
$$

Further, from condition (6) it follows that

$$
d\left(\frac{1}{n} \sum_{k=1}^{n} x_{k}^{q} \frac{\sum_{i=1}^{n} x_{i}-x_{k}}{n-1}-1\right)=0 \Leftrightarrow \frac{1}{n}\left(\sum_{k=1}^{n} q x_{k}^{q-1} \frac{\left(\sum_{i=1}^{n} x_{i}-x_{k}\right)}{n-1}+\sum_{i=1}^{n} x_{i}^{q}-x_{k}^{q}\right) d x_{k}=0 .
$$


It is clear that the resulting equality will be true at the point $\widehat{x}=(1,1, \ldots, 1)$. Thus, we obtain the following relation: $\frac{q+1}{n} \sum_{k=1}^{n} d x_{k}=0$. Hence,

$$
\left.d^{2} L\right|_{N}=\frac{2(n+1)}{n^{2}} q\left(1-q \frac{n-1}{n+1}\right) \sum_{k=1}^{n} d x_{k}^{2}>0, \quad \text { for } \quad 0<q<\frac{n+1}{n-1} .
$$

We prove that for $q>\frac{n+1}{n-1}(n \geq 2)$ there are such $x_{i}^{*}>0(i=1,2, \ldots n)$ that

$$
\left(\frac{1}{n} \sum_{k=1}^{n} x_{k}^{*}\right)^{q+1}<\frac{1}{n} \sum_{k=1}^{n} x_{k}^{*} q \frac{1}{n-1}\left(\sum_{i=1}^{n} x_{i}^{*}-x_{k}^{*}\right) \text {. }
$$

Let $x_{1}^{*}=1+\varepsilon, x_{2}^{*}=x_{3}^{*}=\ldots=x_{n}^{*}=1$, where $\varepsilon \in\left[0 ; \frac{n-1}{q+1}\left(q-\frac{n+1}{n-1}\right)\right)$. Consider the function

$$
F(q, \varepsilon)=\frac{1}{n} \sum_{k=1}^{n} x_{k}^{*} q \frac{1}{n-1}\left(\sum_{i=1}^{n} x_{i}^{*}-x_{k}^{*}\right)-\left(\frac{1}{n} \sum_{k=1}^{n} x_{k}^{*}\right)^{q+1}=1+\frac{(1+\varepsilon)^{q}+\varepsilon-1}{n}-\left(1+\frac{\varepsilon}{n}\right)^{q+1} .
$$

We prove that there is $0<\varepsilon^{*}<\frac{n-1}{q+1}\left(q-\frac{n+1}{n-1}\right)$ such, that $F\left(q, \varepsilon^{*}\right)>0$. Easy to see that $F(q, 0)=0$. Besides,

$$
F_{\varepsilon}^{\prime}(q, \varepsilon)=\frac{1}{n}\left(q(1+\varepsilon)^{q-1}+1-(q+1)\left(1+\frac{\varepsilon}{n}\right)^{q}\right),
$$

thus, $F_{\varepsilon}^{\prime}(q, 0)=0$. Thus, it remains for us to prove that for all $\varepsilon$ of the above interval, the inequality $F_{\varepsilon}^{\prime \prime}(q, \varepsilon)>0$ holds.

Really,

$$
\begin{gathered}
F_{\varepsilon}^{\prime \prime}(q, \varepsilon)=\frac{q(q-1)}{n}(1+\varepsilon)^{q-2}-\frac{q(q+1)}{n^{2}}\left(1+\frac{\varepsilon}{n}\right)^{q-1}= \\
=\frac{q(q+1)}{n^{2}(1+\varepsilon)^{1-q}}\left(\frac{(q-1) n}{(q+1)(1+\varepsilon)}-\left(\frac{1+\frac{\varepsilon}{n}}{1+\varepsilon}\right)^{q-1}\right)>\frac{q(q+1)}{n^{2}(1+\varepsilon)^{1-q}}\left(1-\left(\frac{1+\frac{\varepsilon}{n}}{1+\varepsilon}\right)^{q-1}\right) \geq 0 .
\end{gathered}
$$

Let now $q=\frac{n+1}{n-1}$. The case, when $n=2$ was considered in [1].

Let now $n=3$, then $q=2$. We need to make sure that for non-negative values of variables $x_{1}, x_{2}, x_{3}$ the next inequality will be true: 
Really,

$$
\left(\frac{1}{3} \sum_{k=1}^{3} x_{k}\right)^{3} \geq \frac{1}{3} \sum_{k=1}^{3} x_{k}^{2} \frac{1}{2}\left(\sum_{i=1}^{3} x_{i}-x_{k}\right) .
$$

$$
\begin{aligned}
& \left(\frac{1}{3} \sum_{k=1}^{3} x_{k}\right)^{3}-\frac{x_{1}^{2}\left(x_{2}+x_{3}\right)+x_{2}^{2}\left(x_{1}+x_{3}\right)+x_{3}^{2}\left(x_{1}+x_{2}\right)}{6}= \\
& \frac{1}{27}\left(x_{1}^{3}+x_{2}^{3}+x_{3}^{3}-\frac{1}{2}\left(x_{1} x_{2}\left(x_{1}+x_{2}\right)+x_{1} x_{3}\left(x_{1}+x_{3}\right)+x_{2} x_{3}\left(x_{2}+x_{3}\right)+6 x_{1} x_{2} x_{3}\right) \geq\right. \\
& \geq \frac{2}{9} x_{1} x_{2} x_{3} \geq 0,
\end{aligned}
$$

which follows from the elementary inequality $a^{3}+b^{3} \geq a b(a+b), a \geq 0, b \geq 0$.

Let now $n \geq 4$. Let us prove that there are such $y_{i}>0(i=1,2, \ldots n)$, for which the next inequality is true

$$
\left(\frac{1}{n} \sum_{k=1}^{n} y_{k}\right)^{\frac{2 n}{n-1}}<\frac{1}{n} \sum_{k=1}^{n} y_{k}^{\frac{n+1}{n-1}} \frac{1}{n-1}\left(\sum_{i=1}^{n} y_{i}-y_{k}\right) .
$$

Let $y_{1}=1+\varepsilon, y_{2}=y_{3}=\ldots=y_{n-1}=1, y_{n}=1-\varepsilon$, where $|\varepsilon|<1$. We introduce the function

$$
\begin{aligned}
& F(n, \varepsilon)=\frac{1}{n} \sum_{k=1}^{n} y_{k}^{\frac{n+1}{n-1}} \frac{1}{n-1}\left(\sum_{i=1}^{n} y_{i}-y_{k}\right)-\left(\frac{1}{n} \sum_{k=1}^{n} y_{k}\right)^{\frac{2 n}{n-1}}= \\
& =\frac{1}{n}\left((1+\varepsilon)^{\frac{n+1}{n-1}}\left(1-\frac{\varepsilon}{n-1}\right)+(1-\varepsilon)^{\frac{n+1}{n-1}}\left(1+\frac{\varepsilon}{n-1}\right)-2\right),
\end{aligned}
$$

and prove that there is $0<\left|\varepsilon^{*}\right|<1$ such, that for all $n \geq 4$ the inequality $F\left(n, \varepsilon^{*}\right)>0$ takes place. It is easy to see that $F(n, 0)=0$. Besides,

$$
\begin{gathered}
\left.F_{\varepsilon}^{\prime}(n, \varepsilon)\right|_{\mathcal{E}=0}=\left.\frac{1}{n-1}\left((1+\varepsilon)^{\frac{2}{n-1}}\left(1-\frac{2}{n-1} \varepsilon\right)-(1-\varepsilon)^{\frac{2}{n-1}}\left(1+\frac{2}{n-1} \varepsilon\right)\right)\right|_{\mathcal{E}=0}=0, \\
\left.F_{\mathcal{E}}^{\prime \prime}(n, \varepsilon)\right|_{\mathcal{E}=0}=\left.C(n) \varepsilon\left((1-\varepsilon)^{\frac{3-n}{n-1}}-(1+\varepsilon)^{\frac{3-n}{n-1}}\right)\right|_{\mathcal{E}=0}=0,
\end{gathered}
$$

where $C(n)=\frac{2(n+1)}{(n-1)^{2}}$. It can be directly verified that if $n \geq 4$, then $F_{\varepsilon}^{\prime \prime}(n, \varepsilon)>0$ for $0<|\varepsilon|<1$. As $F_{\mathcal{\varepsilon}}^{\prime}(n, 0)=0$, then function $F_{\mathcal{\varepsilon}}^{\prime}(n, \varepsilon)$ changes sign from «-» on «+» when crossing a point $\varepsilon=0$. It follows that $\min _{|\varepsilon|<1} F(n, \varepsilon)=F(n, 0)=0$. 
Thus, indeed, there is at least one $0<\varepsilon^{*}<1$, for which $F\left(n, \varepsilon^{*}\right)>0$ for $n \geq 4$.

Conclusions. In this paper there is given a generalization of well-known cyclic inequality of N. P. Korneichuk on the case of $n$ independent variables. The proof of this result differs from the proof of the original result in that it required a study on the conditional extremum of the function of $n$ variables, which required very complex calculations and transformations. This result is of independent interest and can be used to obtain estimated results of spline-approximation in classes with bounded modulus of continuity.

\section{Bibliographic references}

1. Корнейчук, Н.П. Сплайны в теории приближения [Текст]/ Н.П. Корнейчук. - М., 1984. $-452 \mathrm{c.}$

2. Алексеев, В.М. Оптимальное управление [Текст] / В.М. Алексеев, В.М. Тихомиров, С.В. Фомин. - М.,1979. - 495 с.

Received 15.10.2019. 